\title{
Absorption Spectra of Solids from Periodic Equation-of-Motion Coupled-Cluster Theory
}

\author{
Xiao Wang ${ }^{1}$ and Timothy C. Berkelbach ${ }^{1,2, *}$ \\ ${ }^{1}$ Center for Computational Quantum Physics, Flatiron Institute, New York, New York 10010 USA \\ ${ }^{2}$ Department of Chemistry, Columbia University, New York, New York 10027 USA
}

\begin{abstract}
We present ab initio absorption spectra of six three-dimensional semiconductors and insulators calculated using Gaussian-based periodic equation-of-motion coupled-cluster theory with single and double excitations (EOM-CCSD). The spectra are calculated efficiently by solving a system of linear equations at each frequency, giving access to an energy range of tens of $\mathrm{eV}$ without explicit enumeration of excited states. We assess the impact of Brillouin zone sampling, for which it is hard to achieve convergence due to the cost of EOM-CCSD. Although our most converged spectra exhibit lineshapes that are in good agreement with experiment, they are uniformly shifted to higher energies by about $1 \mathrm{eV}$. We tentatively attribute this discrepancy to a combination of vibrational effects and the remaining electron correlation, i.e., triple excitations and above.
\end{abstract}

\section{INTRODUCTION}

Absorption spectroscopy is an important tool for studying the electronic properties of materials. For semiconductors and insulators, the low energy part of the absorption spectrum is typically dominated by excitonic effects, which originate from electron-hole interactions that may be screened by the other electrons. The most common computational methods currently used for simulating absorption spectra are timedependent density functional theory (TDDFT) [1-3] and the Bethe-Salpeter equation based on the GW approximation to the self-energy (GW-BSE) [4-8]. In TDDFT, it has long been recognized that the inclusion of nonlocal exchange is critical for the description of excitons [9-12], and promising recent work has applied screened or dielectric-dependent range-separated hybrids [13, 14]. The GW-BSE approach is based on time-dependent many-body perturbation theory and typically includes screening at the level of the randomphase approximation. The predictions are reasonably accurate when compared to experiments, although implementation details, starting point dependence, and the absence of finite-temperature or vibrational effects make a rigorous evaluation challenging. For example, benchmark studies on molecules [15-18] have found that the GW-BSE results depend strongly on the reference functional and the optimal functionals are very different than those typically used for solid-state calculations.

Recent years have seen rapid development of wavefunctionbased quantum chemistry techniques for periodic solids [1925]. In the present context of neutral excitation energies, equation-of-motion coupled-cluster theory with single and double excitations (EOM-CCSD) is a promising alternative to TDDFT or GW-BSE. For example, our group has applied EOM-CCSD to study plasmons in models of metals [26, 27], as well as singly- and doubly-excited states in a molecular crystal [28]. Recently, the two of us presented a systematic study of EOM-CCSD for a range of semiconductors and insulators, finding an accuracy of about $0.3 \mathrm{eV}$ for the first singlet excitation energy [29]. Although these preliminary results are encouraging, the optical response of solids is encoded in the

*tim.berkelbach@gmail.com full energy-dependent absorption spectrum, which depends on all excited states in the energy range of interest and their oscillator strengths. Here, we extend our previous work and study the absorption spectra of semiconductors and insulators predicted by EOM-CCSD.

The remainder of the paper is organized as follows. In Sec. II, we briefly describe the theory underlying the calculation of solid-state absorption spectra with periodic EOMCCSD. In Sec. III, we provide computational details about the basis sets used, integral evaluation, and $k$-point sampling. In Sec. IV, we first present and discuss our final EOM-CCSD optical absorption spectra for six solids, before demonstrating detailed studies of the impact of various approximations. Finally, in Sec, V, we summarize our results and conclude with future directions.

\section{THEORY}

Within EOM-CC theory [30-36], excited states with momentum $\boldsymbol{q}$ are given by

$$
|\Psi(\boldsymbol{q})\rangle=\hat{R}(\boldsymbol{q}) e^{\hat{T}}\left|\Phi_{0}\right\rangle
$$

where $\hat{T}$ creates momentum-conserving particle-hole excitations and $\hat{R}$ creates particle-hole excitations with momentum $\boldsymbol{q}$. The $\hat{T}$ operator is determined by the solution of the nonlinear CC amplitude equations and the $\hat{R}$ operator is determined by a non-Hermitian matrix eigenvalue problem. In crystals, the density of excited states prohibits their direct enumeration. Instead, the absorption (or scattering) spectrum $S_{q}(\omega)$ can be obtained directly at arbitrary frequency by using the solution to a system of linear equations,

$$
\begin{aligned}
& S_{q}(\omega)=-\pi^{-1} \operatorname{Im}\left\langle\Phi_{0}\left|(1+\hat{\Lambda}) \hat{\bar{\mu}}_{q}^{\dagger}\right| x_{q}(\omega)\right\rangle \\
& {\left[\omega-\left(\hat{H}-E_{0}\right)+i \eta\right]\left|x_{q}(\omega)\right\rangle=\hat{\bar{\mu}}_{q}\left|\Phi_{0}\right\rangle .}
\end{aligned}
$$

where $\hat{\bar{O}}=e^{-\hat{T}} \hat{O} e^{\hat{T}}$ are similarity-transformed operators, $\hat{\Lambda}$ is the deexcitation operator needed for expectation values in CC theory, $\hat{\mu}_{q}=\sum_{c v k} \mu_{c k+q, v k} \hat{a}_{c k+q}^{\dagger} \hat{a}_{v k}$ is the transition operator with momentum $\boldsymbol{q}$, and $\eta$ is a numerical Lorentzian linewidth.

Here, we study the performance of EOM-CC with single and double excitations (EOM-CCSD), i.e. $\hat{T}=\hat{T}_{1}+\hat{T}_{2}$, 
$\hat{\Lambda}=\hat{\Lambda}_{1}+\hat{\Lambda}_{2}$, and $\hat{R}=\hat{R}_{1}+\hat{R}_{2}$, and focus on absorption spectra with $\boldsymbol{q}=0$. For each frequency $\omega$, the cost of iteratively solving the system of linear equations (Eq. 2b) scales as $O\left(N_{k}^{4} N_{o}^{2} N_{v}^{4}\right)$, where $N_{k}$ is the number of $k$-points sampled in the Brillouin zone and $N_{o}, N_{v}$ are the number of occupied and virtual orbitals in the unit cell. In practice, the iterative solution converges slowly for some values of $\omega$. Therefore, in this work we also test and apply so-called partitioned EOMCCSD [37-39], where the double excitation block of the similarity transformed Hamiltonian is approximated by a diagonal matrix of orbital energy differences. This reduces the iterative scaling of the EOM step to $O\left(N_{k}^{3} N_{o}^{2} N_{v}^{3}\right)$.

As an alternative to EOM-CC, one can use the linearresponse coupled cluster (LR-CC) theory to calculate excitedstate properties. When no truncation is carried out in the excitation levels, LR-CC and EOM-CC both give exact results. At a truncated excitation level, the methods yield identical excitation energies but different excited-state properties, such as transition dipole moments, and only properties predicted by LR-CC are properly size extensive [40, 41]. Although this finding calls into question the applicability of EOM-CCSD for solid-state absorption spectra, the violation of size extensivity is strongly mitigated when large basis sets are used [42]. In this work, we observe no problems associated with this deficiency of EOM-CCSD for spectra, perhaps because of the near completeness of the basis set in periodic solids.

\section{COMPUTATIONAL DETAILS}

The relatively high cost of periodic CCSD calculations makes it challenging to achieve convergence to the complete basis set and thermodynamic limits. We have tested convergence with respect to Brillouin zone sampling, basis sets, frozen orbitals, and the partioned EOM approximation, which will be discussed in Sec. IV B. Based on our studies, our final calculations presented here are performed in the following way. We use GTH pseudopotentials [43, 44] and the corresponding polarized double-zeta basis set (DZVP) [45]. Twoelectron repulsion integrals were treated by Gaussian density fitting with an even-tempered auxiliary basis (see ref 46 for more details). In the CCSD calculations, we correlate the highest four occupied and the lowest four virtual orbitals at each $k$-point, while all of the other orbitals are frozen. The partitioning approximation is made to the similarity transformed Hamiltonian whereby the dense doubles block is replaced by a diagonal matrix of orbital energy differences [3739]. The Brillouin zone was sampled with a uniform mesh of up to $N_{k}=5 \times 5 \times 5 k$-points. The $k$-point mesh is shifted to include either the $\Gamma$ point or the random symmetry-breaking point $\boldsymbol{k}=(0.11,0.21,0.31)$ (in fractions of the reciprocal lattice vectors). Such random shifts have been previously shown to yield absorption spectra that converge more quickly to the thermodynamic limit [47]. Lastly, we separately treat the convergence of the first excitation energy and extrapolate to the thermodynamic limit by assuming finite-size errors that scale as $N_{k}^{-1}$, as discussed in our previous work [29]. We then rigidly shift the entire absorption spectrum by this finite-size correction, which is $0.1-0.4 \mathrm{eV}$ for the materials and $k$-point meshes considered here. All calculations were performed with PySCF [48, 49].

\section{RESULTS AND DISCUSSION}

\section{A. EOM-CCSD absorption spectrum for six solids}

In Fig. 1, we show our best and final results for the EOMCCSD absorption spectra of six three-dimensional semiconducting and insulating materials: $\mathrm{Si}, \mathrm{SiC}, \mathrm{C}, \mathrm{MgO}, \mathrm{BN}$, and LiF. The experimental lattice constants were used for all systems: $\mathrm{Si}(5.431 \AA), \mathrm{SiC}(4.350 \AA), \mathrm{C}(3.567 \AA), \mathrm{MgO}$ (4.213 $\AA$ ), BN (3.615 $\AA$ ), and $\operatorname{LiF}(4.035 \AA)$. Spectra were obtained using a $5 \times 5 \times 5 \mathrm{k}$-point mesh; in order to give some sense of possible finite-size errors, we show EOM-CCSD results obtained with the two $k$-point shifts mentioned above. Our EOM-CCSD absorption spectra are compared to experimental ones and to those obtained by configuration interaction with single excitations (CIS), which was performed with a denser $7 \times 7 \times 7 k$-point mesh.

As seen in Fig. 1, the EOM-CCSD spectra are in reasonably good agreement with experiment. Different $k$-point shifts give similar spectra for large gap insulators (like $\mathrm{LiF}$ and $\mathrm{BN}$ ) and different spectra for smaller gap semiconductors (like Si and $\mathrm{SiC}$ ), whose main features are shifted from one another by as much as $1 \mathrm{eV}$. When compared to experiment, the EOMCCSD spectra are shifted to higher energies by about $1 \mathrm{eV}$, but otherwise have very similar lineshapes, indicating an accurate description of excitonic interactions and concomitant redistribution of spectral weight. By comparison, CIS spectra massively overestimate the excitation energies of solids by $3 \mathrm{eV}$ or more, as shown in our previous work [29], and often have qualitatively incorrect spectral structure. Because Hartree-Fock-based CIS is identical to unscreened GW-BSE, these results emphasize the well-known importance of screening, especially in semiconductors.

We believe that the shift to higher energies that is exhibited by EOM-CCSD when compared to experiment is mostly attributable to the missing correlation due to the neglect of triple (and higher) excitations and the absence of vibrational and finite-temperature effects, which are of course present in experiments and absent in the calculations. With regards to electron correlation, it is interesting to note that our previous work, which did not study spectra, found that EOM-CCSD overestimated the first excitation energy by about $0.3 \mathrm{eV}$, which is noticably smaller than the deviations seen in the spectra in Fig. 1. This discrepancy (i.e. overestimation by $0.3 \mathrm{eV}$ versus $1 \mathrm{eV}$ or more) is because the first excited state, especially in indirect gap materials, is typically weakly absorbing and contributes to the gradual onset of absorption. However, experimentally reported first excitation energies are typically those of spectral peaks or intense features, which occur at higher energies than the onset of absorption. In Fig. 1, the green vertical lines indicate the calculated first excitation energies, which are corrected for finite-size effects and other approximations mentioned in Sec. III. 

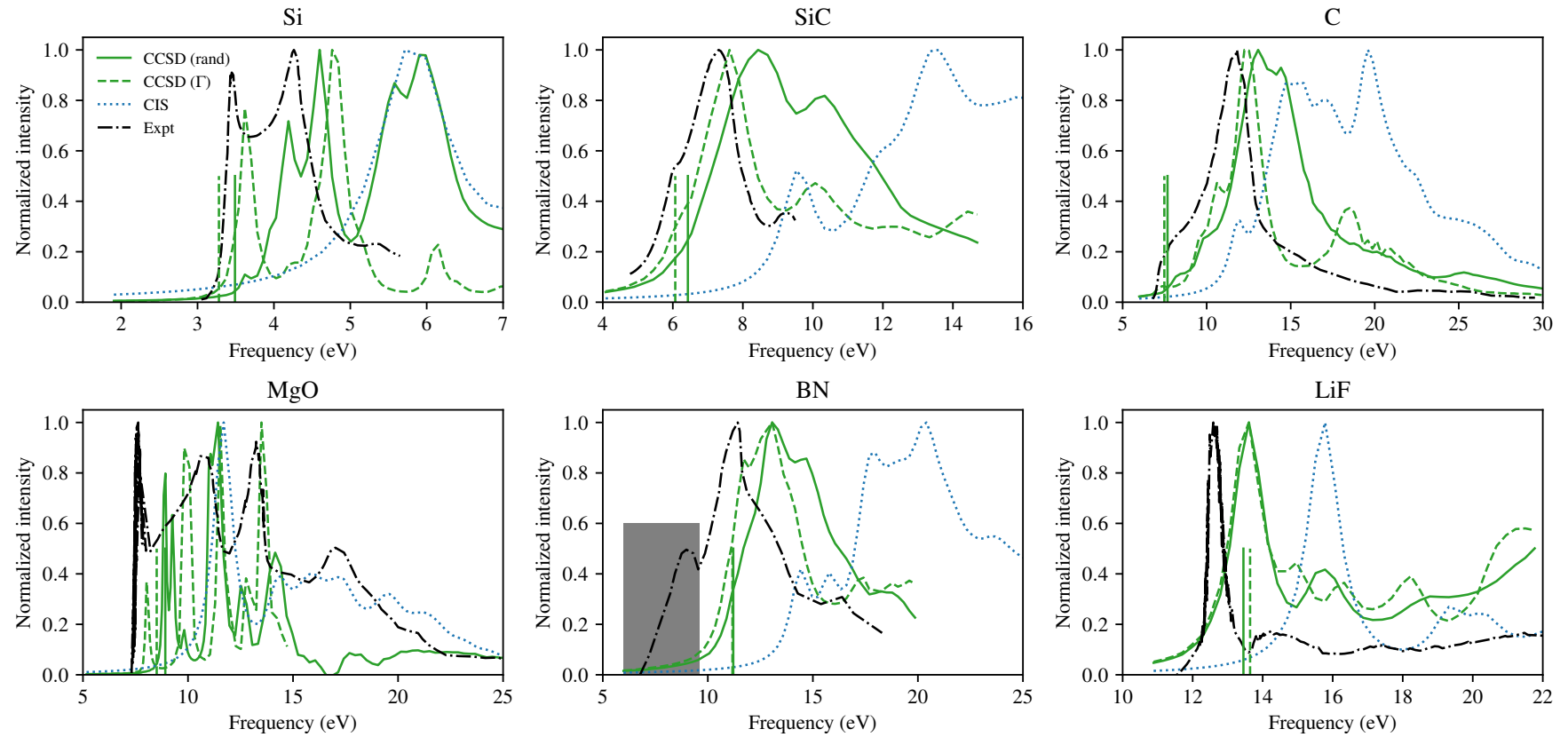

FIG. 1. Absorption spectra of $\mathrm{Si}, \mathrm{SiC}, \mathrm{C}, \mathrm{MgO}, \mathrm{BN}$, and LiF in the DZVP basis set. A $5 \times 5 \times 5 k$-point mesh is used for all CCSD spectra (green) and a $7 \times 7 \times 7 k$-point mesh for all CIS spectra (blue). CCSD spectra are shown with $k$-point meshes that are shifted to include the $\Gamma$ point (dashed green) and a random, symmetry-breaking $k$-point (solid green). The corresponding EOM-CCSD first excitation energies are indicated by green vertical lines. A broadening of $\eta=0.54 \mathrm{eV}$ is used in all calculations except for silicon and $\mathrm{MgO}$ where a smaller broadening of $\eta=0.08 \mathrm{eV}$ is used to resolve the sharp peaks. For BN, the shaded region of the experimental spectrum should be ignored as it has been attributed to defects and polymorphism [50].

In contrast to the apparent differences in spectra, the use of differently shifted $k$-point meshes produce first excitation energies that agree reasonably well with each other, with a difference of $0.04-0.4 \mathrm{eV}$. The first excitation energies differ from our previously reported values [29] (by $0.2 \mathrm{eV}$ or less) due to a slightly different treatment of the finite-size effects in the current work, i.e. (a) we extrapolate the data using a function of the form $E_{\infty}+a N_{k}^{-1}$, (b) here, the $5 \times 5 \times 5$ result is included in extrapolation, and (c) the partitioning approximation is corrected by a constant shift deduced from the $3 \times 3 \times 3$ result. Naturally, we believe that the comparison of spectra, as done here, enables the most fair evaluation of the accuracy. However, this overestimation of excited states by $1 \mathrm{eV}$ or more is significantly higher than the known performance of EOMCCSD in molecules. This difference is surprising, especially given that the excitonic states contributing to absorption are all predominantly single-excitation in character and that the EOM-CCSD polarizability has most of the diagrammatic content of the GW-BSE polarizability, plus more [26, 28, 51, 52].

Among the six solids in Fig. 1, a few show noticeable differences between the EOM-CCSD and experimental spectra. The worst agreement is for silicon, which has the smallest gap of all materials considered. In its experimental spectrum, the main features are the two peaks at $3.5 \mathrm{eV}$ and $4.3 \mathrm{eV}$ with similar intensity. While the CCSD spectra with both $k$-point shifts predict the position of the first peak reasonably well, the randomly shifted $k$-point mesh severely underestimates its intensity relative to that of the higher-lying peaks. In contrast, the $\Gamma$-inclusive $k$-point mesh correctly gives similar intensity for the two-peak structure, although the intensity between the two peaks is strongly underestimated. We believe that the poor agreement between theory and experiment is due to the large remaining finite-size effects, which are expected to be largest for this small-gap semiconductor.

\section{B. Approximations and error corrections}

Finite-size errors of excited-state properties like absorption spectra have been widely discussed in the TDDFT and GWBSE literature [7, 13, 53-55], in part due to the relative maturity and low computational cost of these methods. In constract, the finite-size errors of wavefunction-based methods such as CCSD have been studied signficantly less, especially for spectra. In the following, we will use diamond as an example to study the finite-size errors of the spectra predicted by the EOM-CCSD.

As a warm-up to EOM-CCSD, we first consider CIS, which forms a minimal theory for electronic excited states in the condensed phase and is qualitatively comparable to TDDFT and GW-BSE. Importantly, the relatively low cost of CIS allows us to study the convergence with respect to Brillouin zone sampling up to relatively large $k$-point meshes. In the upper panels of Fig. 2, we show the CIS absorption spectra computed with various $k$-point meshes centered at $\Gamma$ (right column) or randomly shifted (left column), including up to $7 \times 7 \times 7 k$-point 

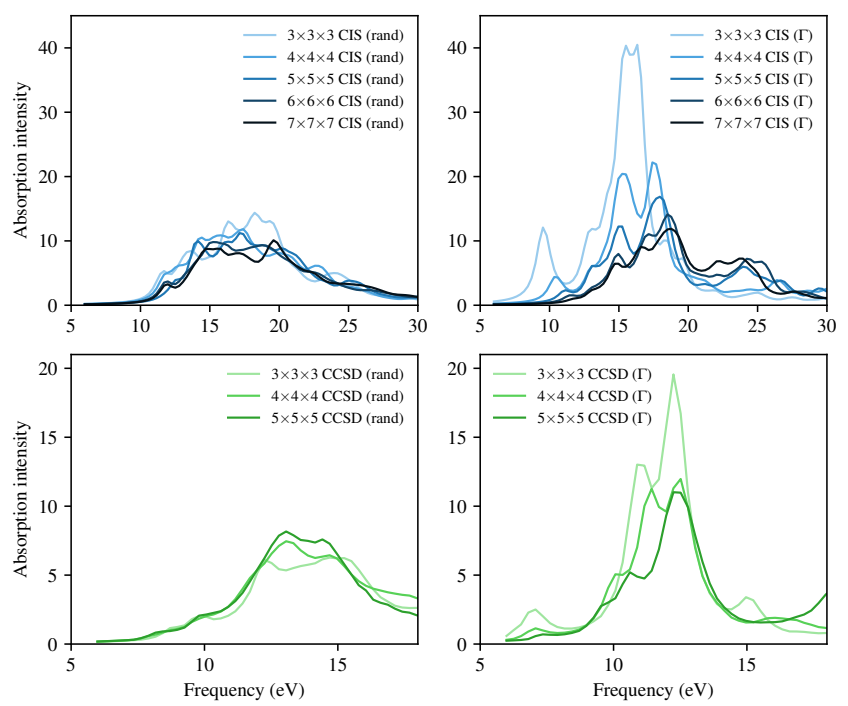

FIG. 2. Convergence of the CIS (top) and EOM-CCSD (bottom) spectra of diamond using various $k$-point shifts and sampling densities.

meshes. At low mesh densities (like $3 \times 3 \times 3$ ), the spectra computed with different $k$-point shifts show a large discrepancy in both peak positions and intensities. This discrepancy is due to insufficient Brillouin zone sampling and largely depends on details of the band structure. As the mesh density is increased, the spectra converge to a similar result, but the convergence is much more rapid with the randomly shifted $k$-point mesh. Even for this insulator, the CIS spectra are not graphically converged with a $7 \times 7 \times 7$ mesh. This must be kept in mind when analyzing the EOM-CCSD spectra in Fig. 1, which are limited to $5 \times 5 \times 5$ meshes.

In the lower panels of Fig. 2, the EOM-CCSD spectra of diamond with the same two $k$-point shifts are shown, for mesh densities ranging from $3 \times 3 \times 3$ to $5 \times 5 \times 5$. As for CIS, we again see that the randomly shifted mesh provides significantly faster convergence towards the thermodynamic limit. In fact, the $4 \times 4 \times 4$ and $5 \times 5 \times 5$ are very similar and suggest semiquantitative convergence, especially at low energies.

In addition to the spectral intensities, the excitation energies also exhibit large finite-size errors. These latter finite-size errors are simpler to remove by extrapolation. Our final EOMCCSD spectra shown in Fig. 1 have been rigidly shifted by the finite-size error of the first excitation energy. This finite-size error is determined by extrapolation, assuming that the finitesize error decays as $O\left(N_{k}^{-1}\right)$. Raw data and extrapolation fits are shown in Fig. 3 for four of the solids studied here. As expected, we see that the convergence is erratic for indirect gap materials ( $\mathrm{C}$ and $\mathrm{Si}$ ) but significantly smoother for direct gap materials ( $\mathrm{LiF}$ and $\mathrm{MgO}$ ). At the largest $k$-point meshes, the finite-size errors are in the range of $0.1-0.4 \mathrm{eV}$.

Beyond the finite-size errors, we have studied the effects of three other approximations: incomplete basis set, frozen orbitals, and the partitioning of EOM-CCSD, as shown in Fig. 4 for diamond with the same randomly-shifted $k$-point mesh as above. In Fig. 4(a), we show that the basis set incompleteness
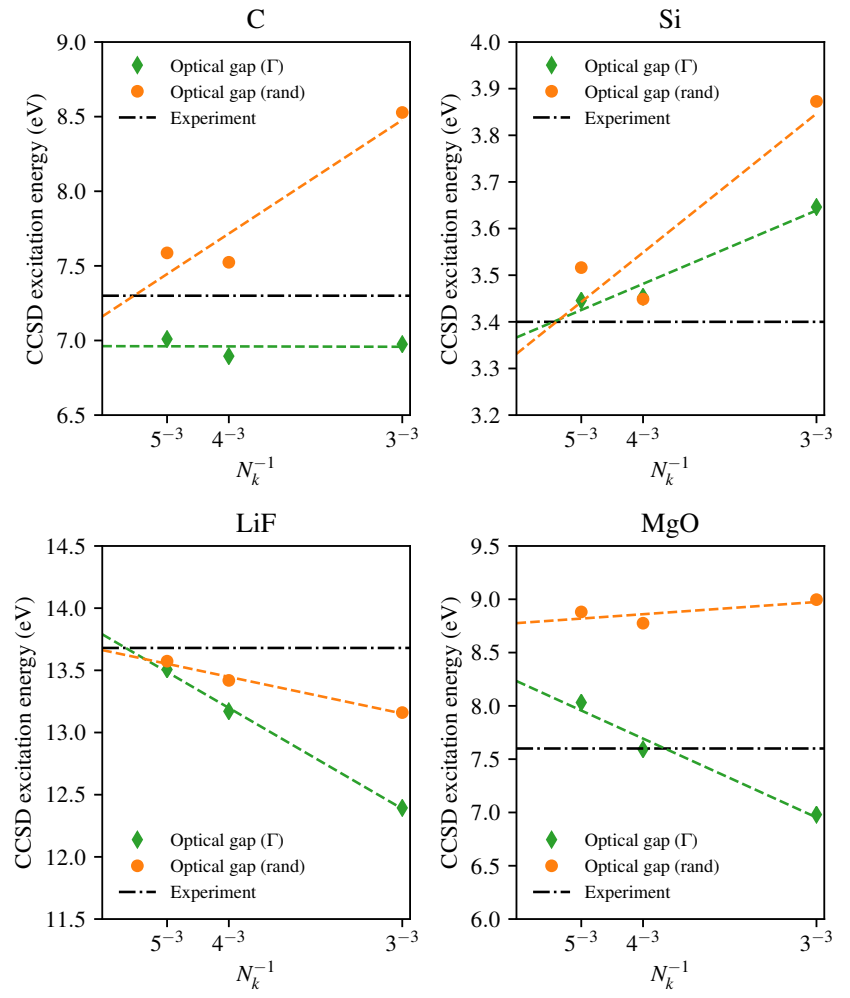

FIG. 3. Extrapolation of first excitation energies to the thermodynamic limit for $\mathrm{C}, \mathrm{Si}, \mathrm{LiF}$, and $\mathrm{MgO}$. Frozen virtual orbitals and partitioning are used in all cases.

error is negligible by comparing the spectrum obtained with two types of pseudopotentials, GTH $[43,44]$ and ccECP [5659], combined with their corresponding double- and triplezeta basis sets. These calculations were performed with a $2 \times 2 \times 2 k$-point mesh and without freezing any orbitals. Additionally, we see that the use of two distinct pseudopotentials does not introduce a noticeable difference in the calculated spectra.

In Fig. 4(b), we test the impact of orbital freezing and partitioning by showing four spectra, all performed with a $3 \times 3 \times 3 k$-point mesh: (1) the EOM-CCSD spectrum without approximations, (2) the EOM-CCSD spectrum with only 4 occupied and 4 virtual orbitals correlated, (3) the partitioned EOM-CCSD spectrum without any frozen orbitals, and (4) the partitioned EOM-CCSD spectrum with 4 occupied and 4 virtual orbitals correlated. We see that freezing orbitals causes a roughly rigid shift of the spectrum to higher energy by about $0.2-0.5 \mathrm{eV}$. The shift is not perfectly rigid and, as expected, the discrepancy is worst at high energies. In contrast, the partitioning error causes a roughly rigid shift to lower energy by a similar amount. When both approximations are applied, we obtain a spectrum close to the one without approximations due to fortuitous cancellation of error, justifying our use of this affordable approach when scaling up to larger $k$-point meshes.

The effect of all errors discussed in this subsection can be approximated with a rigid spectral shift according to the error in the first excitation energy. These corrections for both 

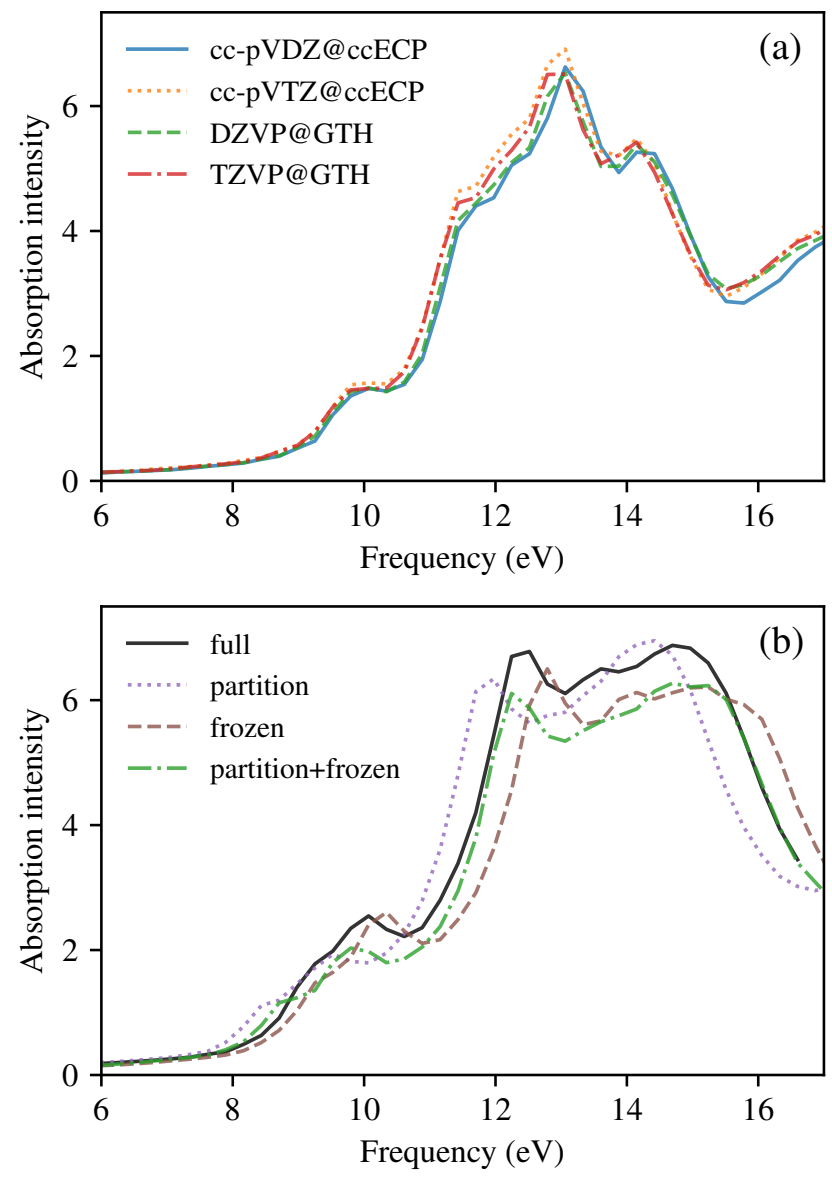

FIG. 4. EOM-CCSD absorption spectra of diamond with various basis sets and approximations as indicated. A random $k$-point shift and a Lorentzian broadening of $\eta=0.54 \mathrm{eV}$ is used in all cases. (a) Comparison of EOM-CCSD spectra (without partitioning and with all orbitals correlated) using four different basis set/pseudopotential combinations as indicated. The Brillouin zone was sampled with a $2 \times 2 \times 2 k$-point mesh. (b) Comparison of the full EOM-CCSD spectra and various approximations as indicated using the DZVP basis set and GTH pseudopotential. The Brillouin zone was sampled with a $3 \times 3 \times 3 k$-point mesh.

$\Gamma$-centered and randomly shifted $k$-point meshes are summarized in Table I for all six material studied. The base result $\left(E_{555}\right)$ is obtained with partitioned EOM-CCSD using frozen orbitals and a $5 \times 5 \times 5 k$-point mesh. To this, we apply two composite-style corrections: $\Delta_{\mathrm{TDL}}$ is the difference between the excitation energy in the thermodynamic limit obtained by extrapolation and $E_{555}$, where all calculations are performed with partitioned EOM-CCSD/DZVP with frozen orbitals, and $\Delta_{\text {frz+part }}$ is the difference between EOM-CCSD without approximations and partitioned EOM-CCSD with frozen orbitals, using a $3 \times 3 \times 3 k$-point mesh. These two corrections are roughly comparable in magnitude but strongly system dependent. Although each correction alone may shift the energy by up to $0.5 \mathrm{eV}$, the final correction is typically quite small due to error cancellation.

To reiterate, the final spectra presented in Fig. 1 were ob-
TABLE I. EOM-CCSD first excitation energies and corrections (in $\mathrm{eV}$ ) for $\mathrm{Si}, \mathrm{SiC}, \mathrm{C}, \mathrm{MgO}, \mathrm{BN}$, and $\mathrm{LiF}$.

\begin{tabular}{lrrrr}
\hline \hline & $E_{555}$ & \multicolumn{1}{c}{$\Delta_{\text {TDL }}$} \\
randomly shifted & $\begin{array}{c}\Delta_{\text {frz }} \text {-point } \\
\text { Si }\end{array}$ & \multicolumn{4}{c}{ mesh } & $E_{\text {final }}$ \\
\cline { 2 - 5 } $\mathrm{SiC}$ & 3.52 & -0.18 & 0.20 & 3.53 \\
$\mathrm{C}$ & 5.83 & 0.14 & 0.53 & 6.50 \\
$\mathrm{MgO}$ & 7.59 & -0.43 & 0.37 & 7.53 \\
$\mathrm{BN}$ & 8.88 & -0.11 & 0.28 & 9.05 \\
$\mathrm{LiF}$ & 11.06 & -0.16 & 0.24 & 11.14 \\
& 13.57 & 0.09 & -0.06 & 13.61 \\
$\mathrm{Si}$ & \multicolumn{5}{r}{$\Gamma$-included } & $k$-point mesh & \\
$\mathrm{SiC}$ & 3.45 & -0.08 & -0.12 & 3.25 \\
$\mathrm{C}$ & 6.10 & -0.28 & 0.24 & 6.06 \\
$\mathrm{MgO}$ & 7.01 & -0.05 & 0.29 & 7.25 \\
$\mathrm{BN}$ & 8.03 & 0.20 & 0.41 & 8.64 \\
$\mathrm{LiF}$ & 10.82 & 0.07 & 0.16 & 11.06 \\
\hline \hline
\end{tabular}

tained with a $5 \times 5 \times 5 k$-point mesh using partitioned EOMCCSD, correlating 4 occupied and 4 virtual orbitals per $k$ point, and then rigidly shifted according to the corrections given in Tab. I to approximately correct for finite-size errors, frozen orbitals, and the partitioning approximation applied to the dense doubles block of the Hamiltonian.

\section{CONCLUSIONS AND OUTLOOK}

We have presented the first absorption spectra of atomistic, three-dimensional solids using periodic EOM-CCSD, focusing on $\mathrm{Si}, \mathrm{SiC}, \mathrm{C}, \mathrm{MgO}, \mathrm{BN}$, and $\mathrm{LiF}$. With increasing Brillouin zone sampling, we observe no problems associated with the lacking size-extensivity of EOM-CCSD spectral intensities $[40,41]$. This may be due to the reasonably complete basis set [42] provided by a solid-state environment, but further study is warranted. After accounting for a number of sources of error, our best and final spectra show reasonably good agreement with experimental spectra, indicating that EOMCCSD is a promising and tractable approach for the study of excitations in solids. In many materials, we find that spectral shapes are well reproduced but are shifted to higher energies with respect to experiment by about $1 \mathrm{eV}$. We attribute this discrepancy to a combination of incomplete electron correlation (i.e., the impact of triple and higher excitations) and the neglect of zero-point and finite-temperature vibrational effects [60-62]. Unlike in TDDFT and GW-BSE, in CCSD there is reduced freedom in the choice of starting point due to its weak sensitivity to the employed reference determinant.

Overall, the agreement between EOM-CCSD and experimental spectra is best for large-gap insulators and worst for small-gap semiconductors, which we attribute to finite-size errors, i.e. incomplete Brillouin zone sampling, and the increasing importance of correlation in small-gap materials. Whereas extrapolation of isolated energies to the thermodynamic limit is largely successful, doing the same for spectral intensities is not straightforward. The high cost of EOM-CCSD calculations precludes brute force convergence and future work will 
explore the use of interpolation [7], twist averaging [63], and double-grid schemes [64], which have been very successful at providing converged GW-BSE spectra at reduced computational cost.

\section{ACKNOWLEDGMENTS}

X.W. thanks Alan Lewis for helpful discussion. This work was supported in part by the National Science Foundation under Grant No. OAC-1931321. All calculations were performed using resources provided by the Flatiron Institute. The Flatiron Institute is a division of the Simons Foundation.
[1] E. Runge and E. K. U. Gross, Phys. Rev. Lett. 52, 997 (1984).

[2] L. Reining, V. Olevano, A. Rubio, and G. Onida, Phys. Rev. Lett. 88, 066404 (2002).

[3] C. A. Ullrich and Z.-h. Yang, "Excitons in time-dependent density-functional theory," in Density-Functional Methods for Excited States, edited by N. Ferré, M. Filatov, and M. HuixRotllant (Springer International Publishing, Cham, 2016) pp. 185-217.

[4] L. J. Sham and T. M. Rice, Phys. Rev. 144, 708 (1966).

[5] W. Hanke and L. J. Sham, Phys. Rev. B 21, 4656 (1980).

[6] S. Albrecht, L. Reining, R. D. Sole, and G. Onida, Phys. Status Solidi A 170, 189 (1998).

[7] M. Rohlfing and S. G. Louie, Phys. Rev. B 62, 4927 (2000).

[8] G. Onida, L. Reining, and A. Rubio, Rev. Mod. Phys. 74, 601 (2002).

[9] F. Bruneval, F. Sottile, V. Olevano, and L. Reining, J Chem Phys 124, 144113 (2006).

[10] S. Botti, A. Schindlmayr, R. D. Sole, and L. Reining, Rep. Prog. Phys. 70, 357 (2007).

[11] J. Paier, M. Marsman, and G. Kresse, Phys. Rev. B 78, 121201 (2008).

[12] A. F. Izmaylov and G. E. Scuseria, J. Chem. Phys. 129, 034101 (2008).

[13] D. Wing, J. B. Haber, R. Noff, B. Barker, D. A. Egger, A. Ramasubramaniam, S. G. Louie, J. B. Neaton, and L. Kronik, Phys. Rev. Materials 3, 064603 (2019).

[14] A. Tal, P. Liu, G. Kresse, and A. Pasquarello, Phys. Rev. Research 2, 032019 (2020).

[15] F. Bruneval, S. M. Hamed, and J. B. Neaton, J. Chem. Phys. 142, 244101 (2015).

[16] D. Jacquemin, I. Duchemin, A. Blondel, and X. Blase, J. Chem. Theory Comput. 12, 3969 (2016).

[17] D. Jacquemin, I. Duchemin, and X. Blase, J. Phys. Chem. Lett. 8, 1524 (2017).

[18] X. Gui, C. Holzer, and W. Klopper, J. Chem. Theory Comput. 14, 2127 (2018).

[19] S. Hirata, I. Grabowski, M. Tobita, and R. J. Bartlett, Chem. Phys. Lett. 345, 475 (2001).

[20] S. Hirata, R. Podeszwa, M. Tobita, and R. J. Bartlett, J. Chem. Phys. 120, 2581 (2004).

[21] H. Katagiri, J. Chem. Phys. 122, 224901 (2005).

[22] A. Grüneis, G. H. Booth, M. Marsman, J. Spencer, A. Alavi, and G. Kresse, J. Chem. Theory Comput. 7, 2780 (2011).

[23] J. McClain, Q. Sun, G. K.-L. Chan, and T. C. Berkelbach, J. Chem. Theory Comput. 13, 1209 (2017).

[24] T. Gruber, K. Liao, T. Tsatsoulis, F. Hummel, and A. Grüneis, Phys. Rev. X 8, 021043 (2018).

[25] I. Y. Zhang and A. Grüneis, Front. Mater. 6 (2019), 10.3389/fmats.2019.00123.

[26] A. M. Lewis and T. C. Berkelbach, Phys. Rev. Lett. 122, 226402 (2019).
[27] B. T. G. Lau and T. C. Berkelbach, J. Chem. Phys. 152, 224704 (2020).

[28] A. M. Lewis and T. C. Berkelbach, J. Phys. Chem. Lett. 11, 2241 (2020).

[29] X. Wang and T. C. Berkelbach, J. Chem. Theory Comput. 16, 3095 (2020).

[30] K. Emrich, Nuclear Physics A 351, 379 (1981).

[31] K. Emrich, Nuclear Physics A 351, 397 (1981).

[32] H. Koch and P. Jørgensen, J. Chem. Phys. 3333-3344, 8059 (1990).

[33] J. F. Stanton and R. J. Bartlett, J. Chem. Phys. 98, 7029 (1993).

[34] R. Bartlett and M. Musiał, Rev. Mod. Phys. 79, 291 (2007).

[35] A. I. Krylov, Annu. Rev. Phys. Chem. 59, 433 (2008).

[36] R. J. Bartlett, WIREs Comput. Mol. Sci. 2, 126 (2012).

[37] M. Nooijen and J. G. Snijders, J. Chem. Phys. 102, 1681 (1995).

[38] J. F. Stanton and J. Gauss, J. Chem. Phys. 103, 1064 (1995).

[39] S. R. Gwaltney, M. Nooijen, and R. J. Bartlett, Chem. Phys. Lett. 248, 189 (1996).

[40] R. Kobayashi, H. Koch, and P. Jørgensen, Chem Phys Lett 219, 30 (1994).

[41] H. Koch, R. Kobayashi, A. Sanchez de Merás, and P. Jo/rgensen, J. Chem. Phys. 100, 4393 (1994).

[42] M. Caricato, G. W. Trucks, and M. J. Frisch, J. Chem. Phys. 131, 174104 (2009).

[43] S. Goedecker, M. Teter, and J. Hutter, Phys. Rev. B 54, 1703 (1996).

[44] C. Hartwigsen, S. Goedecker, and J. Hutter, Phys. Rev. B 58, 3641 (1998).

[45] J. VandeVondele, M. Krack, F. Mohamed, M. Parrinello, T. Chassaing, and J. Hutter, Comput. Phys. Commun. 167, 103 (2005).

[46] Q. Sun, T. C. Berkelbach, J. D. McClain, and G. K.-L. Chan, The Journal of Chemical Physics 147, 164119 (2017).

[47] M. R. Ahmadpour Monazam, K. Hingerl, and P. Puschnig, Phys. Rev. B 88, 075314 (2013).

[48] Q. Sun, T. C. Berkelbach, N. S. Blunt, G. H. Booth, S. Guo, Z. Li, J. Liu, J. D. McClain, E. R. Sayfutyarova, S. Sharma, S. Wouters, and G. K.-L. Chan, WIREs Comput. Mol. Sci. 8, e1340 (2018).

[49] Q. Sun, X. Zhang, S. Banerjee, P. Bao, M. Barbry, N. S. Blunt, N. A. Bogdanov, G. H. Booth, J. Chen, Z.-H. Cui, J. J. Eriksen, Y. Gao, S. Guo, J. Hermann, M. R. Hermes, K. Koh, P. Koval, S. Lehtola, Z. Li, J. Liu, N. Mardirossian, J. D. McClain, M. Motta, B. Mussard, H. Q. Pham, A. Pulkin, W. Purwanto, P. J. Robinson, E. Ronca, E. R. Sayfutyarova, M. Scheurer, H. F. Schurkus, J. E. T. Smith, C. Sun, S.-N. Sun, S. Upadhyay, L. K. Wagner, X. Wang, A. White, J. D. Whitfield, M. J. Williamson, S. Wouters, J. Yang, J. M. Yu, T. Zhu, T. C. Berkelbach, S. Sharma, A. Y. Sokolov, and G. K.-L. Chan, J. Chem. Phys. 153, 024109 (2020). 
[50] A. Tararan, S. di Sabatino, M. Gatti, T. Taniguchi, K. Watanabe, L. Reining, L. H. G. Tizei, M. Kociak, and A. Zobelli, Phys. Rev. B 98, 094106 (2018).

[51] M. F. Lange and T. C. Berkelbach, J. Chem. Theory Comput. 14, 4224 (2018).

[52] T. C. Berkelbach, J. Chem. Phys. 149, 041103 (2018).

[53] R. Laskowski, N. E. Christensen, G. Santi, and C. AmbroschDraxl, Phys. Rev. B 72, 035204 (2005).

[54] F. Fuchs, C. Rödl, A. Schleife, and F. Bechstedt, Phys. Rev. B 78, 085103 (2008).

[55] T. Sander, E. Maggio, and G. Kresse, Phys. Rev. B 92, 045209 (2015).

[56] M. C. Bennett, C. A. Melton, A. Annaberdiyev, G. Wang, L. Shulenburger, and L. Mitas, J. Chem. Phys. 147, 224106 (2017).

[57] M. C. Bennett, G. Wang, A. Annaberdiyev, C. A. Melton, L. Shulenburger, and L. Mitas, J. Chem. Phys. 149, 104108
(2018)

[58] A. Annaberdiyev, G. Wang, C. A. Melton, M. C. Bennett, L. Shulenburger, and L. Mitas, J. Chem. Phys. 149, 134108 (2018).

[59] G. Wang, A. Annaberdiyev, C. A. Melton, M. C. Bennett, L. Shulenburger, and L. Mitas, J. Chem. Phys. 151, 144110 (2019).

[60] J. Noffsinger, E. Kioupakis, C. G. Van de Walle, S. G. Louie, and M. L. Cohen, Phys. Rev. Lett. 108, 167402 (2012).

[61] C. E. Patrick and F. Giustino, J. Phys.: Condens. Matter 26, 365503 (2014).

[62] W. R. L. Lambrecht, C. Bhandari, and M. van Schilfgaarde, Phys. Rev. Materials 1, 043802 (2017).

[63] C. Lin, F. H. Zong, and D. M. Ceperley, Phys. Rev. E 64, 016702 (2001).

[64] D. Kammerlander, S. Botti, M. A. L. Marques, A. Marini, and C. Attaccalite, Phys. Rev. B 86, 125203 (2012). 